\title{
EDITORIAL
}

\section{Impaired muscle mitochondrial density and/or function: a COPD-specific mitochondropathy or simply deconditioning?}

\author{
Aili L. Lazaar* and Paul L. Greenhaff\#
}

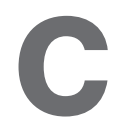

hronic obstructive pulmonary disease (COPD) is a pulmonary disease with some systemic consequences. Skeletal muscle dysfunction is one of the earliest systemic manifestations of COPD in some patients, and contributes to limited exercise capacity and poor health outcomes. The aetiology of skeletal muscle abnormalities in COPD is not fully understood, but probably represents a combination of deconditioning, muscle atrophy and nutritional deficiencies. Studies have shown that skeletal muscle from patients with COPD displays other abnormalities including increased oxidative stress, muscle fibre apoptosis and mitochondrial dysfunction. Indeed, reduced mitochondrial density and function are recognised as important components of skeletal muscle dysfunction in COPD, and reduced oxidative capacity at a skeletal muscle level is likely to contribute to exercise limitation in this population. Mitochondrial density and function are potential therapeutic targets in COPD, but it remains uncertain whether impaired mitochondrial density and/or function are attributable to ageing, inactivity and deconditioning, a COPD-specific "mitochondropathy", or a combination of these factors. However, endurance exercise training is known to increase mitochondrial density and function in healthy volunteers, whether determined by, for example, measurements of maximal activities of mitochondrial enzymes or oxygen consumption and adenosine triphosphate production rates in isolated mitochondria [1]. Given endurance exercise training is an effective intervention strategy in COPD, it stands to reason that such intervention is likely to positively impact upon mitochondrial mass and/or function.

In this issue of the European Respiratory Journal, BRØNSTAD et al. [2] set out to fill some of these gaps in our knowledge. Given that recent research has demonstrated that short-duration, high-intensity interval training is comparable to traditional longer term endurance training in promoting muscle mitochondrial adaptation [3], the authors chose to assess whether undertaking high-intensity unilateral exercise training could stimulate physiological adaptation in COPD patients, the practical rationale here being that the recruitment of localised

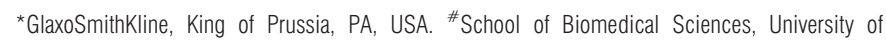
Nottingham Medical School, Nottingham, UK.

CORRESPONDENCE: A.L. Lazaar, GlaxoSmithKline, UW2420, 709 Swedeland Road, King of Prussia, PA 19406, USA. E-mail: aili.I.lazaar@gsk.com muscle groups by using unilateral exercise would reduce the ventilatory limitation to exercise tolerance in COPD patients compared with whole-body exercise, thereby maximising muscle training adaptation. The exercise programme consisted of high-intensity interval knee extensor training over a period of 6 weeks. The authors should be commended for the efforts they took to accurately determine oxygen uptake in the exercising leg.

Importantly, the authors were able to show that high-intensity, unilateral interval training was capable of promoting training adaptation from both a functional and mitochondrial standpoint. However, whether these adaptations were of comparable magnitude to age-matched, healthy control volunteers, and/or greater than conventional endurance-type rehabilitation training in COPD patients was unfortunately not addressed, a limitation that is acknowledged by the authors.

A second valuable observation from the study was that maximal (adenosine diphosphate stimulated) mitochondrial respiration was increased in response to high-intensity, unilateral interval training in COPD patients, and accepting that the study is missing appropriate control data, this does suggest that inactivity rather than mitochondrial dysfunction underlies at least part of exercise intolerance of COPD. The suggestion of the authors that reduced mitochondrial mass, rather than mitochondrial quality, is the key limitation in COPD needs closer scrutiny, particularly as citrate synthase activity (the marker used as an index of mitochondrial mass) was no different between COPD and healthy controls prior to the COPD patients commencing training. This observation of the authors differs from published reports where citrate synthase activity was decreased by up to $40 \%$ in COPD [4,5]. The conclusion that intrinsic abnormalities in mitochondrial respiration do not exist are, however, consistent with previously published data [4]. Finally, the authors observed that maximal mitochondrial respiration was increased without a change in the maximal activity of complex II or IV following exercise. This apparent discrepancy simply may be due to the small number of subjects tested rather than an intrinsic defect, since the trends were similar, but did not reach statistical significance.

Data suggest that acute exercise results in an increase in muscle oxidative stress in patients with COPD, manifested by lipid peroxidation, hydrogen peroxide release and a decrease in mitochondrial DNA [6, 7]. Similarly, others have 
demonstrated that high-intensity endurance training actually depletes glutathione and increases nitrosative stress in patients with COPD [8]. While these observations could be seen to contradict the reported positive effects of endurance exercise training in COPD patients, future studies might examine whether training of an isolated muscle group ameliorates the increase in tissue oxidative stress or modulates the kinetics of the mitochondrial transition pore, another area of uncertainty $[4,9]$.

Pharmacological interventions for the treatment of muscle wasting associated with COPD have been limited. Currently, there are no medications approved by the US Food and Drug Administration for the treatment of muscle wasting of any aetiology. Current approaches to spare or preserve muscle mass and/or function associated with disease and ageing include: increased protein intake; resistance-based exercise programmes; dietary supplements; and off-label use of hormone-replacement therapies. While treatment with anabolic steroids has been shown to increase muscle mass in patients with COPD, the effects on muscle strength and exercise tolerance have been inconsistent. In addition, it has not been demonstrated that increasing muscle mass is an effective mechanism to positively alter muscle mitochondrial function. Novel therapies in development, such as selective androgen receptor modifiers, sirtuin activators and ghrelin mimetics, have the potential to increase muscle mass or stimulate mitochondrial biogenesis, but have not been tested in COPD.

The take-home message, however, is that reducing the ventilatory limitation to exercise training by recruiting small muscle groups may prove to be a valuable strategy in reversing muscle dysfunction and exercise intolerance in COPD. It will also be of importance to determine the effect of this training modality on whole-body exercise tolerance in future work.

\section{STATEMENT OF INTEREST}

A statement of interest for A.L. Lazaar can be found at www.erj. ersjournals.com/site/misc/statements.xhtml

\section{REFERENCES}

1 Wibom R, Hultman E, Johansson M, et al. Adaptation of mitochondrial ATP production in human skeletal muscle to endurance training and detraining. J Appl Physiol 1992; 73: 2004-2010.

2 Brønstad E, Rognmo Ø, Tjonna AE, et al. High-intensity knee extensor training restores skeletal muscle function in COPD patients. Eur Respir J 2012; 40: 1130-1136.

3 Little JP, Safdar A, Wilkin GP, et al. A practical model of low-volume high-intensity interval training induces mitochondrial biogenesis in human skeletal muscle: potential mechanisms. J Physiol 2010; 588: 1011-1022.

4 Picard M, Godin R, Sinnreich M, et al. The mitochondrial phenotype of peripheral muscle in chronic obstructive pulmonary disease: disuse or dysfunction? Am J Respir Crit Care Med 2008; 178: 1040-1047.

5 Puente-Maestu L, Pérez-Parra J, Godoy R, et al. Abnormal mitochondrial function in locomotor and respiratory muscles of COPD patients. Eur Respir J 2009; 33: 1045-1052.

6 Couillard A, Maltais F, Saey D, et al. Exercise-induced quadriceps oxidative stress and peripheral muscle dysfunction in patients with chronic obstructive pulmonary disease. Am J Respir Crit Care Med 2003; 167: 1664-1669.

7 Puente-Maestu L, Lázaro A, Tejedor A, et al. Effects of exercise on mitochondrial DNA content in skeletal muscle of patients with COPD. Thorax 2011; 66: 121-127.

8 Barreiro E, Rabinovich R, Marin-Corral J, et al. Chronic endurance exercise induces quadriceps nitrosative stress in patients with severe COPD. Thorax 2009; 64: 13-19.

9 Puente-Maestu L, Pérez-Parra J, Godoy R, et al. Abnormal transition pore kinetics and cytochrome $\mathrm{C}$ release in muscle mitochondria of patients with chronic obstructive pulmonary disease. Am J Respir Cell Mol Biol 2009; 40: 746-750. 